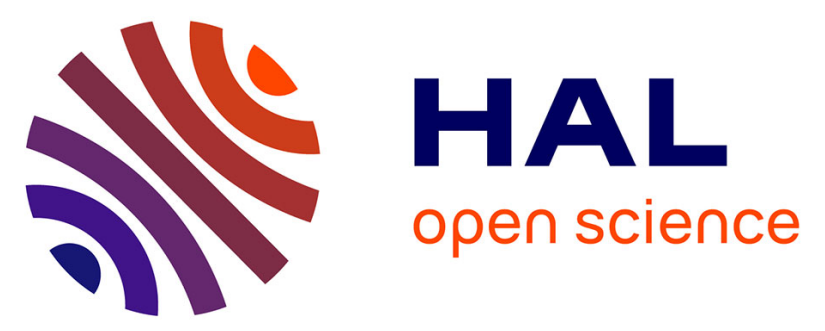

\title{
SLC13A3 variants cause acute reversible leukoencephalopathy and $\alpha$-ketoglutarate accumulation
}

Joseph Dewulf, Elsa Wiame, Imen Dorboz, Monique Elmaleh-Bergès, Apolline A Imbard, Dana Dumitriu, Malgorzata Rak, Agnes Bourillon, Raphaël Helaers, Alisha Malla, et al.

\section{To cite this version:}

Joseph Dewulf, Elsa Wiame, Imen Dorboz, Monique Elmaleh-Bergès, Apolline A Imbard, et al.. SLC13A3 variants cause acute reversible leukoencephalopathy and $\alpha$-ketoglutarate accumulation. Annals of Neurology, 2019, 85 (3), pp.385-395. 10.1002/ana.25412 . hal-02414351

\section{HAL Id: hal-02414351 https://hal.science/hal-02414351}

Submitted on 6 Jan 2021

HAL is a multi-disciplinary open access archive for the deposit and dissemination of scientific research documents, whether they are published or not. The documents may come from teaching and research institutions in France or abroad, or from public or private research centers.
L'archive ouverte pluridisciplinaire $\mathbf{H A L}$, est destinée au dépôt et à la diffusion de documents scientifiques de niveau recherche, publiés ou non, émanant des établissements d'enseignement et de recherche français ou étrangers, des laboratoires publics ou privés. 


\section{$S L C 13 A 3$ variants cause acute reversible leukoencephalopathy and $\alpha K G$ accumulation}

\section{Running head: SLC13A3: reversible leukoencephalopathy and $\alpha$ KG}

Joseph P. Dewulf, $\mathrm{MD}^{1,2,3^{*}}$, Elsa Wiame, $\mathrm{PhD}^{1,2}$, Imen Dorboz, $\mathrm{PhD}^{4}$, Monique ElmalehBergès, $\mathrm{MD}^{5}$, Apolline Imbard PharmD, $\mathrm{PhD}^{6}$, Dana Dumitriu, $\mathrm{MD}^{7}$, Malgorzata Rak, $\mathrm{PhD}^{4}$, Agnès Bourillon ${ }^{6}$, Raphaël Helaers, $\mathrm{PhD}^{8}$, Alisha Malla ${ }^{9}$, Florence Renaldo, $\mathrm{MD}^{4,10,11}$, Odile Boespflug-Tanguy, MD, $\mathrm{PhD}^{4,10,11}$, Marie-Françoise Vincent, $\mathrm{MD}, \mathrm{PhD}^{3}$, Jean-François Benoist, PharmD, $\mathrm{PhD}^{6}$, Ron A. Wevers, $\mathrm{PhD}^{12}$, Avner Schlessinger, $\mathrm{PhD}^{9}$, Emile Van Schaftingen, $\mathrm{MD}, \mathrm{PhD}^{1,2}$, Marie-Cécile Nassogne, $\mathrm{MD}, \mathrm{PhD}^{13}$ and Manuel Schiff, $\mathrm{MD}, \mathrm{PhD}$, $4,10,14^{*}$

${ }^{1}$ Laboratory of Biochemistry, de Duve Institute, UCLouvain, B-1200 Brussels, Belgium

${ }^{2}$ Walloon Excellence in Life sciences and Biotechnology (WELBIO), B-1200 Brussels, Belgium

${ }^{3}$ Department of Laboratory Medicine, Cliniques Universitaires Saint-Luc, UCLouvain B-1200 Brussels, Belgium

${ }^{4}$ UMR1141, PROTECT, INSERM, Université Paris-Diderot, Sorbonne Paris Cité, Paris, France

${ }^{5}$ Department of Pediatric Imaging, Hôpital Universitaire Robert Debré, APHP, Paris, France

${ }^{6}$ Laboratory of Biochemistry, Hôpital Universitaire Robert Debré, APHP, Paris, France; Université Paris Sud, Chatenay Malabry, France

${ }^{7}$ Departement of Pediatric Imaging, Cliniques Universitaires Saint-Luc, UCLouvain, B-1200 Brussels, Belgium ${ }^{8}$ Human Molecular Genetics, de Duve Institute, UCLouvain, B-1200 Brussels, Belgium

${ }^{9}$ Department of Pharmacological Sciences, Icahn School of Medicine at Mount Sinai, New York, NY 10029, USA

${ }^{10}$ Department of Pediatric Neurology and Metabolic Diseases, Hôpital Universitaire Robert Debré, APHP, Paris, France

${ }^{11}$ Reference Center for Leukodystrophies and rare Leukoencephalopathies, LEUKOFRANCE, Hôpital Universitaire Robert Debré, APHP, Paris, France

${ }^{12}$ Translational Metabolic Laboratory, Department of Laboratory Medicine, Radboud University Medical Center, 6500 HB Nijmegen, the Netherlands

${ }^{13}$ Pediatric Neurology Unit, Cliniques Universitaires Saint-Luc, UCLouvain, Brussels, Belgium

${ }^{14}$ Reference Center for Inborn Errors of Metabolism, Hôpital Universitaire Robert Debré, APHP, Paris, France

*Corresponding authors: Joseph Dewulf: joseph.dewulf@uclouvain.be; Manuel Schiff: manuel.schiff@aphp.fr

This article has been accepted for publication and undergone full peer review but has not been through the copyediting, typesetting, pagination and proofreading process which may lead to differences between this version and the Version of Record. Please cite this article as doi: 10.1002/ana.25412

This article is protected by copyright. All rights reserved. 
Keywords: SLC13A3, leukoencephalopathy, alpha-ketoglutarate, N-acetylaspartate, NaDC3 


\begin{abstract}
Objective

SLC13A3 encodes the plasma membrane $\mathrm{Na}^{+} /$Dicarboxylate Cotransporter 3 (NaDC3), which imports inside the cell four to six carbon dicarboxylates as well as N-acetylaspartate (NAA). SLC13A3 is mainly expressed in kidney, in astrocytes and in the choroid plexus. We describe two unrelated patients presenting with acute, reversible (and recurrent in one) neurological deterioration during a febrile illness. Both patients exhibited a reversible leukoencephalopathy and a markedly increased and persisting over time urinary excretion of $\alpha$-ketoglutarate $(\alpha \mathrm{KG})$. In one patient, increased cerebrospinal fluid NAA and dicarboxylates (including $\alpha \mathrm{KG}$ ) concentrations were observed. Extensive workup was unsuccessful and a genetic cause was suspected.
\end{abstract}

\title{
Methods
}

Whole exome sequencing (WES) was performed. Our teams were connected through GeneMatcher.

\section{Results}

WES analysis revealed variants in SLC13A3. A homozygous missense mutation (p.Ala254Asp) was found in the first patient. The second patient was heterozygous for another missense mutation (p.Gly548Ser) and an intronic mutation affecting splicing as demonstrated by RT-PCR performed in muscle tissue (c.1016+3A>G). Mutations and segregation were confirmed by Sanger sequencing. Functional studies performed on HEK293T cells transiently transfected with wild type and mutant SLC13A3 indicated that the missense mutations caused a marked reduction in the capacity to transport $\alpha \mathrm{KG}$, succinate and NAA.

\section{Interpretation}

SLC13A3 deficiency causes acute and reversible leukoencephalopathy with marked accumulation of $\alpha \mathrm{KG}$. Urine organic acids (especially $\alpha \mathrm{KG}$ and NAA) and SLC13A3 mutations should be screened in patients presenting with unexplained reversible leukoencephalopathy for which SLC13A3 deficiency is a novel differential diagnosis. 


\section{INTRODUCTION}

SLC13A3 encodes the plasma membrane $\mathrm{Na}^{+} /$Dicarboxylate Cotransporter 3 (NaDC3), which imports into the cytosol four to six carbon dicarboxylates together with three $\mathrm{Na}^{+}$ions ${ }^{1-3}$. Its main substrates are succinate ${ }^{1,4-7}$, fumarate ${ }^{1,5-7}$, malate ${ }^{1,5,6}$, glutarate ${ }^{1,6,7}, \alpha$-ketoglutarate $(\alpha \mathrm{KG})^{1,5-7}$ and N-acetylaspartate (NAA) ${ }^{8-10}$.

SLC13A3 is mainly expressed in kidney, but also in brain, liver, placenta and eye ${ }^{1,4}$, ${ }^{5,10}$. In the kidney, it is located at the basolateral membrane of proximal tubule cells ${ }^{11,12}$ where it is indirectly involved in the secretion of endogenous or exogenous organic anions by organic anion transporters (OATs). These carriers import organic anions from the interstitial space in exchange with dicarboxylates ${ }^{2,7,13-17}$. In principal cells of connecting and collecting ducts, SLC13A3 is also expressed in the luminal membrane ${ }^{12}$. In the brain, this transporter is present in astrocytes and in the choroid plexus ${ }^{5}$, but absent in neurons ${ }^{18}$.

In this study, we report two individuals from two unrelated families presenting with acute, reversible (and recurrent in one) neurological deterioration in the context of febrile illness. Both patients exhibited a reversible leukoencephalopathy and a markedly increased and persisting over time urinary excretion of $\alpha \mathrm{KG}$.

\section{METHODS}

\section{Whole exome sequencing (WES)}

All procedures conformed to ethical standards. Informed written consent was obtained from the parents of both patients. The ethics committee of Robert Debré University Hospital (APHP, Paris, France) and of the Faculty of Medicine of UCLouvain (Brussels, Belgium) approved the genetic and molecular studies on patient's samples.

For patient $1(\mathrm{P} 1)$, library preparation, exome capture, sequencing and data analysis were performed by IntegraGen SA (Evry, France) as previously described ${ }^{19}$. For patient 2 (P2), exome sequencing and analysis with help of the Highlander software were performed essentially as in $^{20}$. The retained variants: (i) passed GATK standard quality-control filters; (ii) co-segregated with the disease as expected for a recessive transmission; (iii) induced missense or nonsense mutations, or altered consensus splice-site; (iv) showed a $<1 \%$ allele 
frequency in the ExAC database (http://exac.broadinstitute.org/); (v) were not detected in inhouse databases of 201 (P1) and 699 (P2) WES; (vi) (for missense variants) were predicted to affect protein function by $\geq 3$ out of 6 tools: SIFT, LRT, Mutation Assessor, FATHMM Mutation Taster and Polyphen2.

\section{cDNA analysis}

RNA was extracted from muscle of P2 and three control muscles using TriPure isolation reagent (Roche). PCR-amplifications from cDNAs were carried out with GoTAQ DNA polymerase (Promega) using forward 5'-TGG CCA GCT CAA GAG TTT CT-3' and reverse 5'-TGT GTT GGG AGC TTT GAA GTC-3' primers.

\section{Cloning and site-directed mutagenesis}

All PCR-amplifications were carried out with Q5 High-Fidelity DNA polymerase (New England Biolabs). SLC13A3 was amplified from human kidney cDNA with forward primer 5'-AT CGA ATT CCC ACC ATG GCG GCG CTG GCA GCA GCG-3' and reverse primer 5'-CAG AAG CTT TCA GAG GGT CCG AAA TGT GTC ATT G-3'. The resulting amplified fragment was digested with EcoRI and HindIII and inserted between the corresponding restriction sites of plasmid pCMV5. The resulting construct was checked by sequencing and corresponded to GenBank accession AAH35966.1. Mutations were introduced by site-directed mutagenesis and checked by sequencing.

\section{Transfection of HEK-293T cells, deglycosylation assay and western blot analysis}

HEK293T cells were grown in high glucose DMEM supplemented with 10\% heat-inactivated fetal bovine serum, $2 \mathrm{mM}$ UltraGlutamine and antibiotics (100 units/ml penicillin and 100 $\mu \mathrm{g} / \mathrm{ml}$ streptomycin) at $37^{\circ} \mathrm{C}$ in $5 \% \mathrm{CO}_{2}$. Twenty-four hours before transfection, cells were seeded in 12 well plates and 6 well plates at 1.5 or $4 \times 10^{5}$ cells/plate for uptake experiments and deglycosylation assays, respectively. SLC13A3 constructs or empty pCMV5 vector were transfected using the jetPEI ${ }^{\mathrm{TM}}$ procedure (Polyplus transfection). After incubation for $48 \mathrm{~h}$ at $37^{\circ} \mathrm{C}$, cells were used for dicarboxylate uptake (see below) or deglycosylation assays. PNGaseF (NEB) and EndoH (Roche) treatments were performed on intact cells and on cell 
lysates essentially as described previously ${ }^{21}$. After the in vivo deglycosylation, cells were directly collected in 1 x concentrated reducing sample buffer. Cell lysates and western blots were performed as previously described ${ }^{22}$ using rabbit anti-SLC13A3 antibody (ab56017, Abcam) diluted 1:1000 in PBS containing $1 \%$ bovine serum albumin. Detection was performed with ImageQuant LAS4000 (GE Healthcare).

\section{Dicarboxylic acids and $\mathrm{N}$-acetylaspartate uptake assays}

$\left[1,4-{ }^{14} \mathrm{C}\right]$ Succinate was from Moravek. Radiolabeled NAA was prepared by acetylation of $\left[\mathrm{U}-{ }^{14} \mathrm{C}\right]$ aspartate (Moravek) with acetic anhydride, and radiolabeled $\alpha \mathrm{KG}$, from $[\mathrm{U}-$ ${ }^{14}$ C]glutamate (Perkin-Elmer) through an exchange reaction catalyzed by glutamateoxaloacetate transaminase in the presence of a 50-fold excess of unlabeled $\alpha \mathrm{KG}$. Their radiochemical purity was $>98 \%$ and $\approx 95 \%$ as estimated by anion exchange chromatography. Our protocol for transport assays was adapted from ${ }^{23}$. Briefly, cells were washed once with a buffer containing $10 \mathrm{mM}$ Hepes, $\mathrm{pH}$ 7.4, $140 \mathrm{mM} \mathrm{NaCl}, 2 \mathrm{mM} \mathrm{KCl}, 1$ $\mathrm{mM} \mathrm{MgCl} 2,1 \mathrm{mM} \mathrm{CaCl} 2$ and $5 \mathrm{mM}$ glucose. Then, $0.1 \mu \mathrm{Ci}\left[1,4-{ }^{14} \mathrm{C}\right]$ succinate, $\left[\mathrm{U}_{-}{ }^{14} \mathrm{C}\right] \alpha \mathrm{KG}$ or $\left[1-4-{ }^{14} \mathrm{C}\right] \mathrm{NAA}$ were added together with the indicated concentrations of unlabeled substrate to initiate the assay. After 5 and $15 \mathrm{~min}$ at $37^{\circ} \mathrm{C}$, the medium was removed and the cells rapidly washed twice with $2 \mathrm{ml}$ ice-cold buffer; $0.2 \mathrm{ml} 1 \mathrm{M} \mathrm{NaOH}$ was added to lyse the cells, followed by $0.25 \mathrm{ml} 1 \mathrm{M} \mathrm{HCl}$. The resulting extract was mixed with $5 \mathrm{ml}$ Scintillation cocktail and its radioactivity counted. Accumulated radioactivity at $15 \mathrm{~min}$ was about 3 times higher than at $5 \mathrm{~min}$, indicating that the uptake measurements were performed in the linear phase.

\section{The SLC13A3 homology model}

A previously published homology model of SLC13A3 was used ${ }^{23}$. The model was based on the $\mathrm{Na}^{+}$-dependent dicarboxylate transporter from Vibrio cholerae (VcINDY) ${ }^{24}$. The two proteins are members of the divalent anion: $\mathrm{Na}+$ symporter (DASS) family, sharing sequence identity of $33 \%$ as well as key functional motifs (e.g., the SNT motifs). The coordinates of the $\mathrm{Na}^{+}$ions and of succinate were derived from a higher resolution structure of a humanized 
variant of VcINDY ${ }^{25}$ (PDB identifier 5UL7) by structurally aligning the SLC13A3 model and the new VcINDY structure with PyMOL.

\section{RESULTS}

\section{Patient 1 (P1):}

This boy, second child out of three, born to consanguineous parents (Fig 1) had no medical history and exhibited normal growth and development. At 15 years and 5 months, 2 days after a febrile $\left(39^{\circ} \mathrm{C}\right)$ respiratory tract infection (treatment: amoxi-clav and oxomemazin), he was admitted for acute neurological deterioration including drowsiness, dysarthria and ataxia. Clinical examination was normal apart from Glasgow coma scale of 8-10/15. Blood glucose, ammonia, electrolytes, liver function tests, CRP, blood cell count and bacterial cultures (blood and urines), toxic and drug screen were non-contributive. CSF analysis showed no increased cell count, slightly elevated protein concentration $(50 \mathrm{mg} / \mathrm{dL}$; normal: $15-45)$, and no oligoclonal band. Brain MRI showed bilateral and symmetric signal abnormalities of the white matter (WM) in the periventricular regions and in the centrum semiovale, and signal abnormalities in the corpus callosum (Fig 2). WM appeared hypointense in T1-weighted axial views (Fig 2A and 2D) and hyperintense in FLAIR axial views (Fig 2B and 2E). ADC (apparent diffusion coefficient) map through the lateral ventricles (Fig 2C) and the centrum semiovale (Fig 2F) showed restricted diffusion in the WM including the corpus callosum. Brain magnetic resonance spectroscopy (MRS) showed normal NAA and lactate peaks. Metabolic workup disclosed increased urinary excretion mainly of $\alpha \mathrm{KG}$ but also of NAA, succinate and fumarate. These organic acids were also found increased in the CSF (Table S1). Citrate and lactate were normal both in the CSF and urine.

After 12 hours of intravenous glucose and electrolytes, the patient recovered a normal clinical status. Brain MRI performed 12 days later disclosed almost complete regression of the WM abnormalities (Fig 2 G-L). Later on, the patient never exhibited any relapse of similar symptoms. He was seen at the outpatient clinic one month, 7 years, and 10 years after the initial episode with normal clinical status and examination. There was persistent abnormal urinary excretion of the dicarboxylates (mainly $\alpha \mathrm{KG}$, Table S1). Brain MRI performed 7 
years after the initial episode was deemed normal (not shown) and showed very mild FLAIR hyperintensities of the WM comparable with what had been observed 12 days after the initial acute event (Fig $2 \mathrm{H}$ and $2 \mathrm{~K}$ ).

\section{Patient 2 (P2):}

This first child of non-consanguineous parents (Fig 1) had normal growth and development. She exhibited febrile seizures at 2 and 4 years of age for which a brain CT scan and electroencephalogram were normal. At 5 years and 8 months, she presented with febrile tonsillitis and was treated with oral amoxicillin. Twenty-four hours later, fever persisted at $39.5^{\circ} \mathrm{C}$ and she exhibited an acute neurological deterioration compatible with encephalitis. On admission, she presented with drowsiness, dysarthria, poor contact, global hypotonia and abnormal peripheral movements. Laboratory parameters (including toxic screen, glucose, ammonia, CRP, urine and blood cultures) were non-contributive. CSF analysis showed increased cell count $\left(84 / \mathrm{mm}^{3}\right.$ [normal range: 0-5], mainly neutrophils), protein of $40 \mathrm{mg} / \mathrm{dL}$ and moderate elevation of lactate (3.5 mM [normal: 1.1-2.2 $\mathrm{mM}$ ]) but no oligoclonal band. Extensive bacterial and viral workup excluded a known cause of infectious meningoencephalitis. Semi-quantitative ketones testing in urine was positive (4+). Brain MRI showed bilateral, symmetric signal abnormalities of the WM in the periventricular regions and the centrum semiovale, and signal abnormalities in the corpus callosum (Fig 3). WM appeared hypointense in T1-weighted axial views (Fig 3A and 3D) and hyperintense in FLAIR axial views (Fig 3B and 3E). ADC map through the lateral ventricles (Fig 3C) and the centrum semiovale (Fig 3F) showed restricted diffusion in the WM including the corpus callosum. Brain MRS showed normal NAA and lactate peaks. At variance with P1, there were also cerebellar abnormalities with FLAIR hyperintensities in the middle cerebellar peduncles (Fig 3H) with restricted diffusion (Fig 3I). Spine MRI was normal (not shown). Metabolic workup showed a markedly increased urinary excretion of $\alpha \mathrm{KG}$ on repeated samples (Table $\mathrm{S} 1$ ).

The patient was initially treated with intravenous acyclovir, ceftriaxone and methylprednisolone bolus ( $1 \mathrm{~g} / 1.73 \mathrm{~m}^{2} /$ day on 3 consecutive days). After a few days, there was an almost complete recovery apart from cerebellar ataxia. Brain MRI performed 15 days 
after the initial episode disclosed almost complete regression of the WM abnormalities (Fig 3J-M) including those of the middle cerebellar peduncles (Fig 3O). Brain MRI performed one year later confirmed the almost complete regression of the supra-tentorial WM abnormalities but disclosed cerebellar atrophy (Fig 3P and 3Q), consistent with persistence of mild cerebellar ataxia and dysmetria. Six years later, after a 2-day history of fever $\left(39.6^{\circ} \mathrm{C}\right)$, the patient exhibited acute onset ataxia and agitation with cerebellar signs. She was started on i.v. ceftriaxone and acyclovir. Brain CT scan was normal (not shown) and MRI was not performed. CSF analysis showed normal results (cell count, protein, glucose, oligoclonal band). Urine organic acids were not performed. She recovered over a few days. Nine years after the first acute event, clinical follow-up was satisfactory apart from mild cerebellar signs as sole persisting clinical abnormalities. The high excretion of $\alpha \mathrm{KG}$ in urine was persistent (Table S1).

\section{Whole exome sequencing and impact of the potential splicing mutation}

Analysis of WES data from the patients and their families led to the identification of variants in SLC13A3. P1 was homozygous for c.761C $>$ A (p.Ala254Asp) and P2 was compound heterozygous for c.1642G $>$ A (p.Gly548Ser) and c.1016+3A $>$ G. Variants and segregations

were verified by sequencing. Our teams were connected through GeneMatcher ${ }^{26}$. To verify that the mutation c. $1016+3 \mathrm{~A}>\mathrm{G}$ (at the border of exon 7 and intron 7) affected splicing, we PCR-amplified cDNA derived from skeletal muscle, the only available tissue from P2. Using primers located in exon 5 and 9, we obtained one PCR product of expected size (450 bp) in muscle cDNA from 3 controls, but 3 bands of $\approx 450, \approx 350$ and $\approx 250$ bp from P2's cDNA. Sequencing indicated that these products corresponded to normally spliced sequence for the largest product; to an exon-7 missing cDNA for the intermediate-size product; and to an exon-7- and exon-8-missing sequence for the smallest product (Fig 4). These findings indicated that the identified mutation significantly affected splicing.

\section{Effect of the mutations on SLC13A3 expression, glycosylation and function}


No expression of SLC13A3 could be detected in fibroblasts from P1 by cDNA amplification and western blot. The effect of the missense mutations on the expression and the function of SLC13A3 were therefore evaluated in transiently transfected HEK-293T. western blot analysis with anti-SLC13A3 antibody showed two bands with apparent molecular masses of 50 and $45 \mathrm{kDa}$ and a fainter doublet of $\approx 60 \mathrm{kDa}$ with the wild type and the mutated proteins, and no signal in cells transfected with an empty plasmid (Fig 5, upper panel). Treatment of cell extracts with PNGaseF and EndoH (which remove mature and immature N-glycans, respectively), indicated that the $50 \mathrm{kDa}$ band was sensitive to both enzymes, while the 60 $\mathrm{kDa}$ doublet was only digested with PNGaseF. This indicated that the doublet corresponded to mature forms of SLC13A3, while the $50 \mathrm{kDa}$ band contained immature forms of N-glycans and the $45 \mathrm{kDa}$ band was not glycosylated. Remarkably, neither of the two mutations affected the expression of the three main forms of SLC13A3. Treatment of intact transfected cells with PNGaseF showed that the $60 \mathrm{kDa}$ doublet was digested (Fig. 5, lower panel), which indicated that the mature form of SLC13A3 was reachable by the glycosidase without cell disruption and was therefore located in the plasma membrane ${ }^{21}$. Logically, this doublet was not affected by EndoH treatment.

Functional assays with radiolabeled substrates showed that expression of wild type SLC13A3 allowed the cells to take up radiolabeled succinate, $\alpha \mathrm{KG}$ and NAA, while this was not the case in cells transfected with an empty plasmid (Fig. 6). Transfection with the mutated plasmids indicated that the proteins bearing the mutations A254D and G548S had a reduced transport capacity. Kinetic analysis indicated that mutation G548S markedly reduced the transport Vmax but decreased the Km value for all three substrates, while mutation A254D almost abolished the transport activity (Fig.6 and Table S2). It was therefore not possible to calculate kinetic parameters for this mutant.

\section{Mutation effects on SLC13A3 structure and function}

In an attempt to explain the effect of the mutations on the function of SLC13A3, we used the published dimer model of SLC13A3 ${ }^{28}$ and derived the coordinates of the sodium atoms and the substrate succinate from a new VcINDY structure ${ }^{25}$. The model contains eleven 
transmembrane helices (TM1-TM11), two helix-turn-helix hairpins (HPin and HPout), a substrate molecule (succinate), and two of the three predicted sodium ions ( $\mathrm{Na} 1$ and $\mathrm{Na} 2$ ) (Fig 7). Notably, the SLC13 family members are thought to transport via an "elevator" mechanism, where the domain containing the substrate (transport domain) moves along the axis perpendicular to the membrane, and oligomerization domain is static ${ }^{27}$.

Molecular modeling of the variants suggested that Ala254 is located in TM5b making up part of the substrate-binding site. Mutation of Ala254 to the acidic aspartate is expected to significantly affect the shape and electrostatic potential of the binding site, thereby likely preventing SLC13A3 interaction with anionic substrates. Ala254 is also located in the interface between the transport and the oligomerization domains; thus, newly introduced aspartate may hinder helix-helix packing and proper movement of transport domain, which is required for substrate translocation. Modeling of the Gly548 variant suggested that it is located in TM11, within the alpha-helix bundle of the transport domain. Mutation of this glycine residue to serine might therefore impact SLC13A3 structure and dynamics. Glycine has been found to play a role in helix-helix interactions and a mutation of this residue would most likely decrease the flexibility of the helix, disrupt helix-helix packing, and disrupt ligand transport ${ }^{28}$. This would be consistent with the markedly reduced Vmax observed with this mutant. Regarding the variant affecting the splicing in P2, exon 7 skipping should result in the deletion of the loop between TM6 and TM7, while exon 8 skipping should result in the deletion of TM7 and the majority of the loop between TM7 and TM8. It is likely that the removal of such considerable part of the transmembrane region of the protein may affect overall folding, stability, and dynamics.

\section{DISCUSSION}

The two patients with biallelic SLC13A3 mutations exhibited a similar phenotype of reversible abnormalities both at the clinical and neuroimaging level. One of them (P1) exhibited a single acute episode while the other had a relapse 6 years after a first acute episode and subsequently showed a persisting mild cerebellar syndrome. 
Metabolic analyses pointed to a permanent increase of urinary $\alpha \mathrm{KG}$ concentration in both patients. Different causes of $\alpha \mathrm{KG}$ accumulation could be excluded. Dihydrolipoamide dehydrogenase and $\alpha \mathrm{KG}$ oxidation assays were performed on fibroblasts of P1 and all exons of $D L D, O G D H, D L S T$ and $O G D H L$ (encoding subunits of $\alpha \mathrm{KG}$ dehydrogenase) were sequenced for P2. These tests were normal. Defects in thiamine transport or metabolism ${ }^{29,30}$ were also ruled out by analyzing WES data. High urinary excretion of $\alpha \mathrm{KG}$ is associated with D-2- and L-2-hydroxyglutaric aciduria in mitochondrial citrate transporter deficiency ${ }^{31}$, but no abnormal excretion of 2-hydroxyglutaric acid was noted in either of the patients.

Functional analysis indicated that the SLC13A3 mutations found in the patients markedly reduced the capacity of this protein to transport the three tested substrates and the effects on the kinetic properties of the transporter were consistent with the location and the putative function of the mutated residues as suggested by a homology model. Tests with glycosidases indicated that the mutations did not grossly affect the glycosylation status and suggested that the decrease in transport induced by the mutations was not due to a decreased localization in the plasma membrane (at least with the overexpression system that we used), but to a change in the intrinsic transport capacity of the carrier. We may not exclude that trafficking defects may also contribute to decreased functionality of the mutants under more physiological conditions. Surprisingly, the missense mutation found in P2 (p.Gly548Ser) affected less the activity of the transporter than the missense mutation found in P1 (p.Ala254Asp), despite the fact that P2 was clinically more severely affected than P1. Nonetheless, $\mathrm{P} 2$ is not homozygous for this missense mutation and therefore the comparison is difficult to interpret.

SLC13A3 is mainly present at the basolateral membrane of cells in the proximal tubule and its described function is to import dicarboxylates from the interstitial space ${ }^{12}$. It was therefore rather unexpected to find high amounts of $\alpha \mathrm{KG}$ in urine of patients deficient in this transporter. However, a moderate staining with the SLC13A3 antibody has also been described at the basolateral and apical membranes in principal cells of connecting segments and collecting ducts ${ }^{12}$. The main organic anion transporters (OAT1 and OAT3) were not detected in these locations ${ }^{11,12}$. Thus, in the distal nephron, SLC13A3 might be involved in 
the reabsorption of dicarboxylates from the glomerular filtrate and its inactivation might therefore explain increased excretion of dicarboxylic acids in the two patients.

$\alpha \mathrm{KG}$ was the most abundant dicarboxylate found in urine of both patients and was permanently elevated. Urinary succinate, fumarate, glutarate and NAA were also found to be abnormally high and persisting over time in P1. For P2, glutarate was inconsistently found above the normal limit and an abnormal peak of NAA was also found. The finding that the predominant dicarboxylate found in urine is $\alpha \mathrm{KG}$ and not succinate is possibly due to the fact that succinate is also well transported by $\mathrm{NaDC} 1$ (SLC13A2), another transporter present in the kidney, which is much less efficient to transport $\alpha \mathrm{KG}^{2,32,33}$. Thus, SLC13A3 may be the major transporter involved in the reuptake of $\alpha \mathrm{KG}$, while there would be redundancy of transporters for succinate.

Analysis performed in P1 (but not in P2) indicated that the concentration of NAA, $\alpha \mathrm{KG}$ and other dicarboxylic acids were elevated in the CSF. This is likely the consequence of lack of functioning of SLC13A3 in its two localizations in the CNS: the epithelial cells of the choroid plexus and the astrocytes. Data obtained on apical membrane vesicles derived from the epithelium of the bovine choroid plexus indicate that the $\mathrm{Na}$ /dicarboxylate carrier (presumably SLC13A3) pumps dicarboxylates from the CSF to the cytosol and creates a transmembrane gradient that can be used by OATs to extract organic acids from the CSF and secrete them in the blood ${ }^{5,34-36}$. This role is analogous to that exerted in the epithelial cells of the kidney primary tubule, except that SLC13A3 and OAT1 are expressed at the basolateral membrane, and serve therefore for the transfer of organic anions from blood to urine ${ }^{2}$. Though we have no data to support this, we speculate that decreased functionality of SLC13A3 may obliterate the urinary excretion and the elimination from the CSF of organic anions, possibly xenobiotics, and that this may have played a role in the crises observed in the two patients.

The physiological function of SLC13A3 in astrocytes is unclear. A major potential substrate is NAA, which is made in neurons from aspartate and acetyl-CoA by NAT8L ${ }^{37}$ and destroyed by aspartoacylase, an enzyme, which according to studies in rodents, is mainly present in oligodendrocytes, but not in astrocytes ${ }^{38}$. Due to its location, there is therefore no 
obvious role for SLC13A3 in NAA metabolism. Loss of function of ASPA leads to Canavan disease $^{39}$, a severe spongiform leukodystrophy with accumulation of NAA in brain tissue and body fluids, detectable as an increased NAA peak on MRS ${ }^{40}$. Absence of increase in NAA peak in SLC13A3 deficiency reflects the much lower accumulation of NAA, which is consistent with the idea that SLC13A3 is not on the main pathway leading from NAA synthesis in the neurons to NAA degradation in oligodendrocytes.

Like in other inborn errors of metabolism affecting the CNS, and particularly glutaric aciduria type I, a triggering event seems to be necessary to cause decompensation and the appearance of lesions ${ }^{41}$. In the present case, the triggering event may have been a fever episode, which indeed preceded the three acute neurological episodes experienced by the two patients. How fever eventually leads to decompensation is unknown. Yet it is probably wise to recommend controlling temperature by appropriate measures and avoiding catabolic states in SLC13A3 defective patients.

In conclusion, SLC13A3 deficiency causes a marked hyperexcretion of $\alpha \mathrm{KG}$ in urine and favors the development of an acute, reversible leukoencephalopathy. Urine organic acids (especially $\alpha \mathrm{KG}$ and NAA) and SLC13A3 mutations should be screened in patients with unexplained leukoencephalopathies. This will most likely lead to the identification of additional patients and to a better definition of the clinical picture of this novel disease. 


\title{
ACKNOWLEDGEMENTS
}

This study was supported by the Fonds National de la Recherche Scientifique (FNRS), the Walloon Excellence in Life sciences and Biotechnology (WELBIO), the European Leukodystrophy Association, grant number ELA 2009-007I4 and by the European Union FP7 RD Connect project. This work was supported in part by a grant from the National Institutes of Health (R01 GM108911 to A.S. and A.M.). We thank the patients and their families for participation in this study. We thank Dr Hélène Ogier de Baulny for providing clinical data on P1, Dr Sandrine Marie for providing clinical laboratory data on P2, and Dr Alexandra Ntorkou for her help in generating the brain imaging figures.

\author{
AUTHOR CONTRIBUTIONS \\ JPD, EW, MFV, EVS, MCN, and MS contributed to conception and design of the study; \\ JPD, EW, ID, MEB, AI, DD, MR, AB, RH, AM, FR, OBT, MFV, JFB, RAW, AS, EVS, \\ $\mathrm{MCN}$, and MS contributed to acquisition of the data and data analysis; JPD, EW, ID, RH, \\ FR, AS, EVS, MCN and MS drafted the text or prepared figure.
}

\section{POTENTIAL CONFLICTS OF INTEREST}

None. 


\section{REFERENCES}

1. Wang H, Fei YJ, Kekuda R, et al. Structure, function, and genomic organization of human $\mathrm{Na}(+)$-dependent high-affinity dicarboxylate transporter. Am J Physiol Cell Physiol 2000;278:C1019-1030.

2. Pajor AM. Sodium-coupled dicarboxylate and citrate transporters from the SLC13 family. Pflugers Arch 2014;466:119-130.

3. Bergeron MJ, Clemencon B, Hediger MA, Markovich D. SLC13 family of Na(+)coupled di- and tri-carboxylate/sulfate transporters. Mol Aspects Med 2013;34:299-312.

4. Kekuda R, Wang H, Huang W, et al. Primary structure and functional characteristics of a mammalian sodium-coupled high affinity dicarboxylate transporter. J Biol Chem 1999;274:3422-3429.

5. Pajor AM, Gangula R, Yao X. Cloning and functional characterization of a highaffinity $\mathrm{Na}(+)$ /dicarboxylate cotransporter from mouse brain. Am J Physiol Cell Physiol 2001;280:C1215-1223.

6. Burckhardt BC, Lorenz J, Kobbe C, Burckhardt G. Substrate specificity of the human renal sodium dicarboxylate cotransporter, $\mathrm{hNaDC}-3$, under voltage-clamp conditions. Am J Physiol Renal Physiol 2005;288:F792-799.

7. Kaufhold M, Schulz K, Breljak D, et al. Differential interaction of dicarboxylates with human sodium-dicarboxylate cotransporter 3 and organic anion transporters 1 and 3. Am J Physiol Renal Physiol 2011;301:F1026-1034.

8. Huang W, Wang H, Kekuda R, et al. Transport of N-acetylaspartate by the $\mathrm{Na}(+)-$ dependent high-affinity dicarboxylate transporter $\mathrm{NaDC} 3$ and its relevance to the expression of the transporter in the brain. J Pharmacol Exp Ther 2000;295:392-403.

9. Fujita T, Katsukawa H, Yodoya E, et al. Transport characteristics of N-acetyl-Laspartate in rat astrocytes: involvement of sodium-coupled high-affinity carboxylate transporter NaC3/NaDC3-mediated transport system. J Neurochem 2005;93:706-714.

10. George RL, Huang W, Naggar HA, Smith SB, Ganapathy V. Transport of Nacetylaspartate via murine sodium/dicarboxylate cotransporter $\mathrm{NaDC} 3$ and expression of this transporter and aspartoacylase II in ocular tissues in mouse. Biochim Biophys Acta 2004;1690:63-69.

11. Bai X, Chen X, Feng Z, et al. Identification of basolateral membrane targeting signal of human sodium-dependent dicarboxylate transporter 3. J Cell Physiol 2006;206:821-830.

12. Breljak D, Ljubojevic M, Hagos Y, et al. Distribution of organic anion transporters NaDC3 and OAT1-3 along the human nephron. Am J Physiol Renal Physiol 2016;311:F227238.

13. Shimada H, Moewes B, Burckhardt G. Indirect coupling to $\mathrm{Na}+$ of p-aminohippuric acid uptake into rat renal basolateral membrane vesicles. Am J Physiol 1987;253:F795-801.

14. Pritchard JB. Coupled transport of p-aminohippurate by rat kidney basolateral membrane vesicles. Am J Physiol 1988;255:F597-604.

15. Shuprisha A, Lynch RM, Wright SH, Dantzler WH. Real-time assessment of alphaketoglutarate effect on organic anion secretion in perfused rabbit proximal tubules. Am $\mathrm{J}$ Physiol 1999;277:F513-523. 
16. Hagos Y, Krick W, Braulke T, Muhlhausen C, Burckhardt G, Burckhardt BC. Organic anion transporters OAT1 and OAT4 mediate the high affinity transport of glutarate derivatives accumulating in patients with glutaric acidurias. Pflugers Arch 2008;457:223-231. 17. Pritchard JB. Intracellular alpha-ketoglutarate controls the efficacy of renal organic anion transport. J Pharmacol Exp Ther 1995;274:1278-1284.

18. Yodoya E, Wada M, Shimada A, et al. Functional and molecular identification of sodium-coupled dicarboxylate transporters in rat primary cultured cerebrocortical astrocytes and neurons. J Neurochem 2006;97:162-173.

19. Dorboz I, Aiello C, Simons C, et al. Biallelic mutations in the homeodomain of NKX6-2 underlie a severe hypomyelinating leukodystrophy. Brain 2017;140:2550-2556.

20. Basha M, Demeer B, Revencu N, et al. Whole exome sequencing identifies mutations in $10 \%$ of patients with familial non-syndromic cleft lip and/or palate in genes mutated in well-known syndromes. J Med Genet 2018;55:449-458.

21. Straumann N, Wind A, Leuenberger T, Wallimann T. Effects of N-linked glycosylation on the creatine transporter. Biochem J 2006;393:459-469.

22. Wiame E, Tahay G, Tyteca D, et al. NAT6 acetylates the N-terminus of different forms of actin. FEBS J 2018;285:3299-3316.

23. Schlessinger A, Sun NN, Colas C, Pajor AM. Determinants of substrate and cation transport in the human $\mathrm{Na}+$ /dicarboxylate cotransporter NaDC3. J Biol Chem 2014;289:16998-17008.

24. Mancusso R, Gregorio GG, Liu Q, Wang DN. Structure and mechanism of a bacterial sodium-dependent dicarboxylate transporter. Nature 2012;491:622-626.

25. Nie R, Stark S, Symersky J, Kaplan RS, Lu M. Structure and function of the divalent anion/ $\mathrm{Na}(+)$ symporter from Vibrio cholerae and a humanized variant. Nat Commun 2017;8:15009.

26. Sobreira N, Schiettecatte F, Valle D, Hamosh A. GeneMatcher: a matching tool for connecting investigators with an interest in the same gene. Hum Mutat 2015;36:928-930.

27. Mulligan C, Fenollar-Ferrer C, Fitzgerald GA, et al. The bacterial dicarboxylate transporter VcINDY uses a two-domain elevator-type mechanism. Nat Struct Mol Biol 2016;23:256-263.

28. Colas C, Pajor AM, Schlessinger A. Structure-Based Identification of Inhibitors for the SLC13 Family of $\mathrm{Na}(+)$ /Dicarboxylate Cotransporters. Biochemistry 2015;54:4900-4908. 29. Ortigoza-Escobar JD, Alfadhel M, Molero-Luis M, et al. Thiamine deficiency in childhood with attention to genetic causes: Survival and outcome predictors. Ann Neurol 2017;82:317-330.

30. Sgobbi de Souza PV, Bortholin T, Vieira de Rezende Pinto WB, Bulle Oliveira AS. Teaching NeuroImages: An extremely rare cause of treatable acute encephalopathy. Neurology 2016;87:e116.

31. Nota B, Struys EA, Pop A, et al. Deficiency in SLC25A1, encoding the mitochondrial citrate carrier, causes combined D-2- and L-2-hydroxyglutaric aciduria. Am J Hum Genet 2013;92:627-631.

32. Wright SH, Kippen I, Klinenberg JR, Wright EM. Specificity of the transport system for tricarboxylic acid cycle intermediates in renal brush borders. J Membr Biol 1980;57:7382. 
33. Yao X, Pajor AM. The transport properties of the human renal $\mathrm{Na}(+)$ - dicarboxylate cotransporter under voltage-clamp conditions. Am J Physiol Renal Physiol 2000;279:F54-64.

34. Pritchard JB, Sweet DH, Miller DS, Walden R. Mechanism of organic anion transport across the apical membrane of choroid plexus. J Biol Chem 1999;274:33382-33387.

35. Burckhardt G. Drug transport by Organic Anion Transporters (OATs). Pharmacol Ther 2012;136:106-130.

36. Nigam SK, Bush KT, Martovetsky G, et al. The organic anion transporter (OAT) family: a systems biology perspective. Physiol Rev 2015;95:83-123.

37. Wiame E, Tyteca D, Pierrot N, et al. Molecular identification of aspartate Nacetyltransferase and its mutation in hypoacetylaspartia. Biochem J 2009;425:127-136.

38. Moffett JR, Arun P, Ariyannur PS, Garbern JY, Jacobowitz DM, Namboodiri AM. Extensive aspartoacylase expression in the rat central nervous system. Glia 2011;59:14141434.

39. Matalon R, Michals K, Sebesta D, Deanching M, Gashkoff P, Casanova J. Aspartoacylase deficiency and $\mathrm{N}$-acetylaspartic aciduria in patients with Canavan disease. Am J Med Genet 1988;29:463-471.

40. Jakobs C, ten Brink HJ, Langelaar SA, et al. Stable isotope dilution analysis of Nacetylaspartic acid in CSF, blood, urine and amniotic fluid: accurate postnatal diagnosis and the potential for prenatal diagnosis of Canavan disease. J Inherit Metab Dis 1991;14:653-660.

41. Goodman SI, Frerman FE. In The metabolic and molecular bases of inherited disease. $8^{\text {th }}$ ed. Scriver et al. eds. MacGraw-Hill; 2001: pp. 2195-2204.

42. Wevers RA, Engelke U, Wendel U, de Jong JG, Gabreels FJ, Heerschap A. Standardized method for high-resolution 1H-NMR of cerebrospinal fluid. Clin Chem 1995;41:744-751. 


\section{Figure and table legends}

\section{Figure 1: Pedigrees of the families and identified mutations}

$\mathrm{P} 1$ is homozygous for mutation c.761C $>\mathrm{A}$ (p.Ala254Asp) and P2 is compound heterozygous for mutation c. $1642 \mathrm{G}>\mathrm{A}$ (p.Gly548Ser) and the splicing mutation c.1016+3A $>\mathrm{G}$.

\section{Figure 2: Brain magnetic resonance imaging of $P 1$}

(A-F) MRI during the acute phase. T1-weighted axial view through the lateral ventricles (A) and the centrum semiovale (D) showing white matter (WM) hypointensity, including the corpus callosum. FLAIR axial view through the lateral ventricles (B) and the centrum semiovale (E) showing WM hyperintensity, including the corpus callosum. ADC (apparent diffusion coefficient) map through the lateral ventricles $(\mathrm{C})$ and the centrum semiovale $(\mathrm{F})$ showing restricted diffusion in the WM, including the corpus callosum.

(G-L) MR imaging at the recovery phase (12 days later). Axial views at the same location: T1 $(\mathrm{G}, \mathrm{J})$ showing clear regression of the WM hypointensity with near-normal signal apart from mild hypointensity in the posterior periventricular regions. FLAIR $(\mathrm{H}, \mathrm{K})$ showing clear regression of the WM hyperintensity, which has almost disappeared. Normal $\operatorname{ADC} \operatorname{map}(\mathrm{I}, \mathrm{L})$.

\section{Figure 3: Brain magnetic resonance imaging of P2}

(A-F) MRI during the acute phase. T1-weighted axial view through the lateral ventricles (A) and the centrum semiovale (D) showing periventricular WM hypointensity, including the corpus callosum. FLAIR axial view through the lateral ventricles (B) and the centrum semiovale (E) showing WM hyperintensity, including the corpus callosum. ADC map through the lateral ventricles $(\mathrm{C})$ and the centrum semiovale $(\mathrm{F})$ showing restricted diffusion, including the corpus callosum.

(J-M) MR imaging at the recovery phase (15 days later). Axial views at the same location: T1 $(\mathrm{J}, \mathrm{L})$ showing clear regression of the WM hypointensity with near-normal 
signal. FLAIR (K, M) showing clear regression of the WM hyperintensity. Diffusion weighted sequence with ADC mapping was not performed.

(G-I, N-Q) Cerebellar involvement in P2. T1-weighted axial view through the cerebellum during the acute phase $(\mathrm{G})$ and 15 days later $(\mathrm{N})$ showing normal aspect; FLAIR axial view through the cerebellum during the acute phase showing hyperintensity of the proximal middle cerebellar peduncles (MCP) $(\mathrm{H})$, which has almost disappeared 15 days later $(\mathrm{O})$. ADC map through the cerebellum at the acute phase showing restricted diffusion in the proximal MCP (I). One year later, development of a cerebellar atrophy both on T1-weighted sagittal view (P) and FLAIR axial view (Q).

\section{Figure 4: Splicing defect in patient 2 cDNA.}

cDNA prepared from skeletal muscle of P2 and 3 controls was PCR-amplified and the amplification products analyzed by agarose gel electrophoresis (upper panel). One band of $\approx$ $450 \mathrm{bp}$ was amplified in the controls (first 3 lanes: $\mathrm{C} 1$ to $\mathrm{C} 3$ ) and 3 bands of size $\approx 450 \mathrm{bp}$ $(\mathrm{A}), \approx 350 \mathrm{bp}(\mathrm{B})$ and $\approx 250 \mathrm{bp}(\mathrm{C})$ were found in the case of P2 (last lane). Sequencing indicated that band A corresponds to the normally spliced sequence while bands B and C lack exon 7 and exon 7 and 8, respectively (see lower panel). The positions of the forward and reverse primers are indicated. MW: Molecular weight.

\section{Figure 5: Expression of SLC13A3 in transfected HEK293T cells.}

HEK293T cells were transfected with empty pCMV5 vector (Empty), or pCMV5 vectors encoding wild type (WT) or mutated (A254D; G548S) SLC13A3. Upper panel: cell lysates were prepared, incubated for $1 \mathrm{~h}$ with PNGaseF or EndoH and analyzed by western blotting with anti-SLC13A3 antibodies. Lower panel: intact cells were incubated for $1 \mathrm{~h}$ with PNGaseF or EndoH. Cell extracts were prepared and analyzed by western blotting. Arrows indicate forms of SLC13A3 with mature N-glycans (doublet), mixed type N-glycans and nonglycosylated forms. Each lane was loaded with $50 \mu \mathrm{g}$ protein. The blots are representative of at least three blots performed on independent experiments. 
Figure 6: $\alpha$-Ketoglutarate, succinate and N-acetylaspartate uptake in HEK293T cells transfected with wild type or mutated SLC13A3.

Cells transfected with the indicated plasmids (see Fig. 5) were incubated for 15 min at $37^{\circ} \mathrm{C}$ with the radiolabeled substrates and the indicated concentrations of unlabeled substrates. Substrate uptake is computed as the amount for $1.5 \times 10^{5}$ seeded cells. $\mathrm{K}_{\mathrm{M}}$ and $\mathrm{V}_{\max }$ (table S2) were computed from the data after subtraction of a blank corresponding to the radioactivity accumulated in the control condition (empty vector). Results shown are means \pm SD for 3 independent experiment.

\section{Figure 7: Homology model of SLC13A3.}

Side (A) and cytosolic (B) views of the predicted SLC13A3 dimeric structure with a close view of the binding site and mutated residues (C). The substrate (succinate; orange) and the mutated residues (A254 and G548; yellow and blue, respectively) are shown as sticks, where oxygen and nitrogen atoms are displayed in red and blue, respectively. Sodium ions (Na1 and $\mathrm{Na} 2)$ are displayed as purple spheres. The transmembrane helices are colored based on the respective domains, including the transport domain (pink), the static domain (teal), and the oligomerization domain (blue); loop regions are colored in gray. 


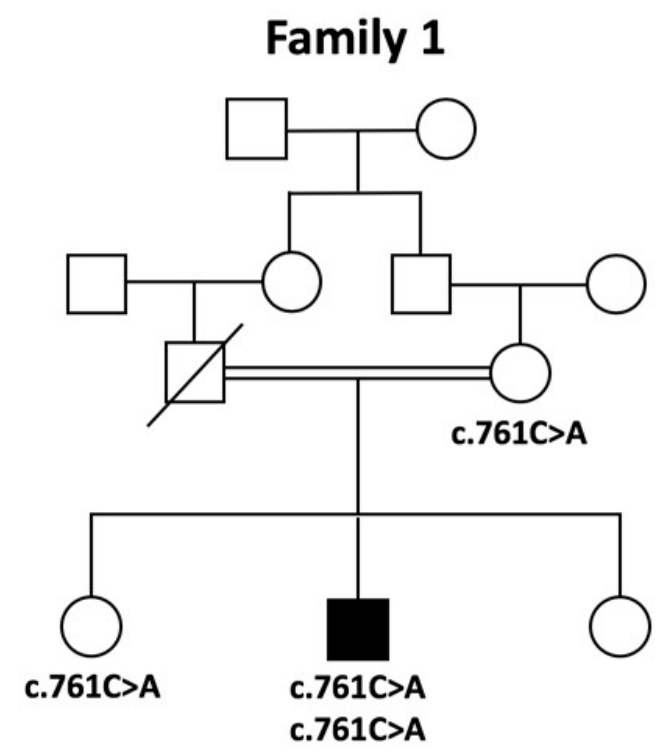

\section{Family 2}

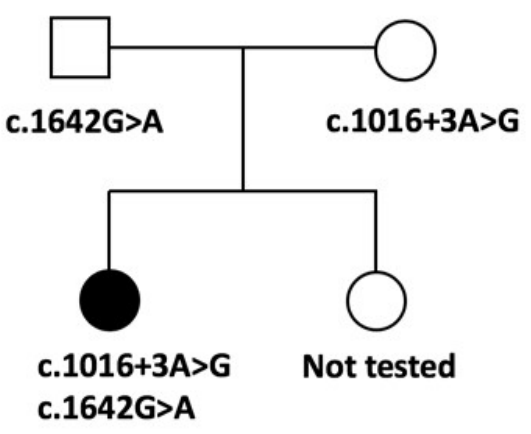

This article is protected by copyright. All rights reserved. 

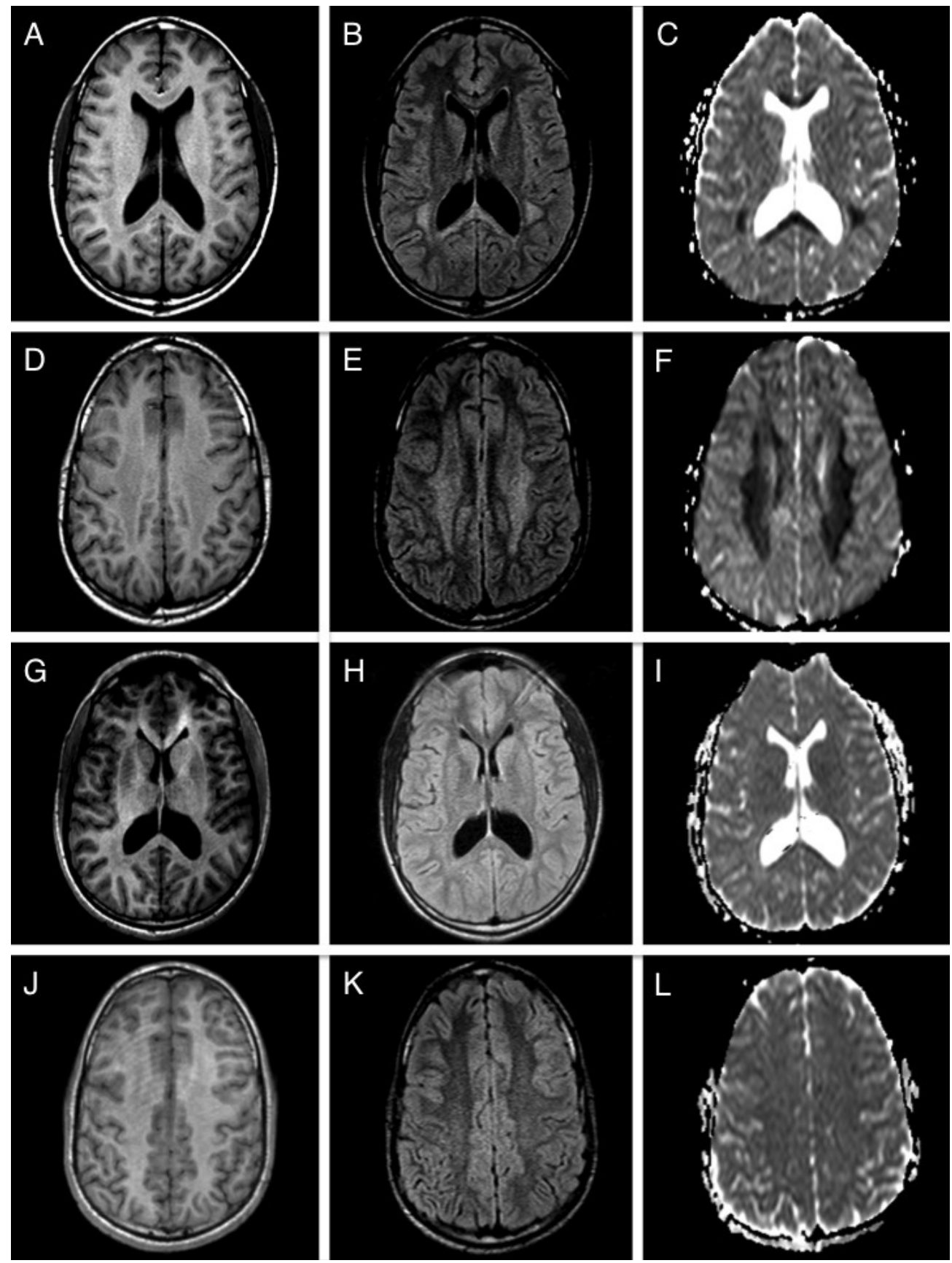

Figure 2 
Figure 3
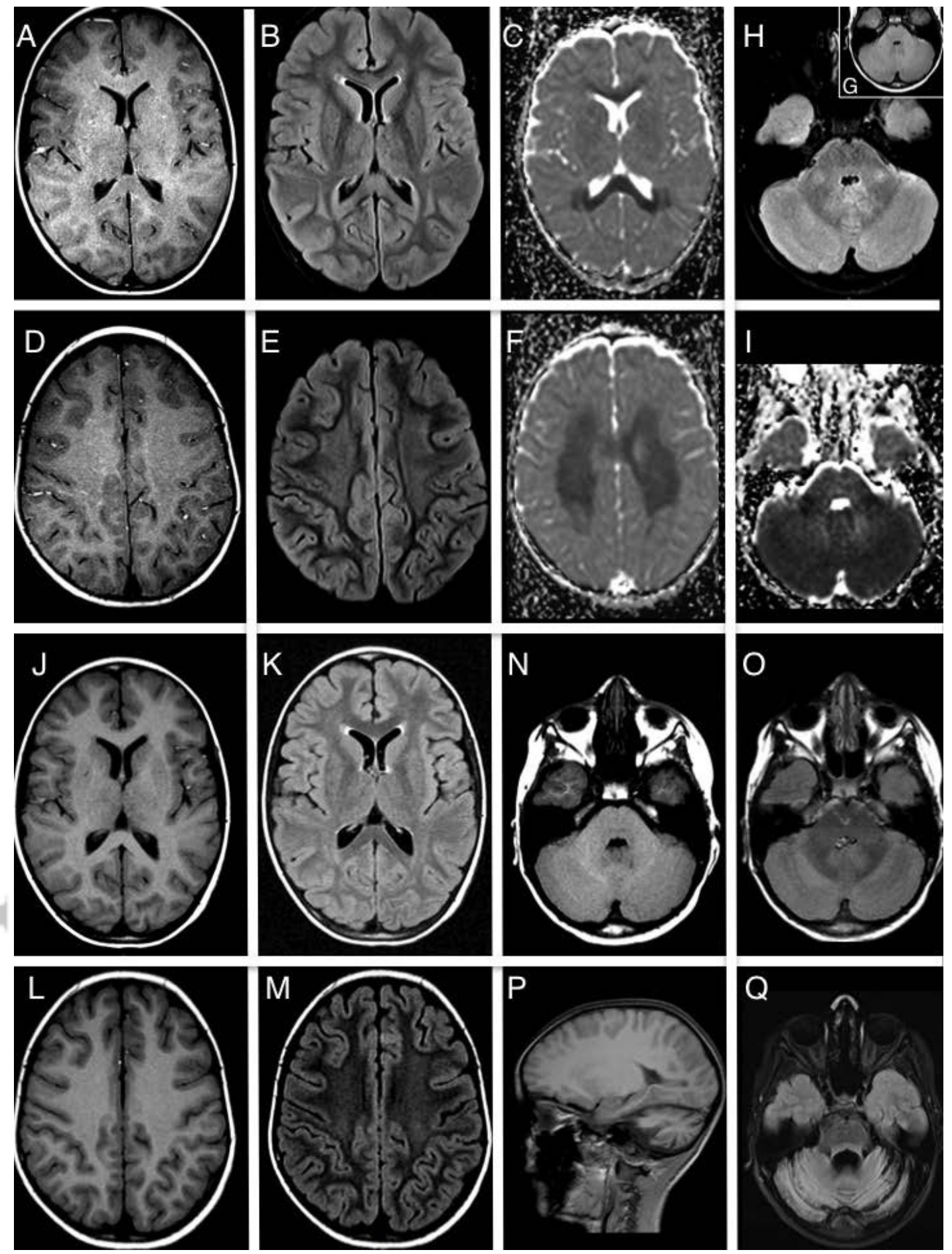


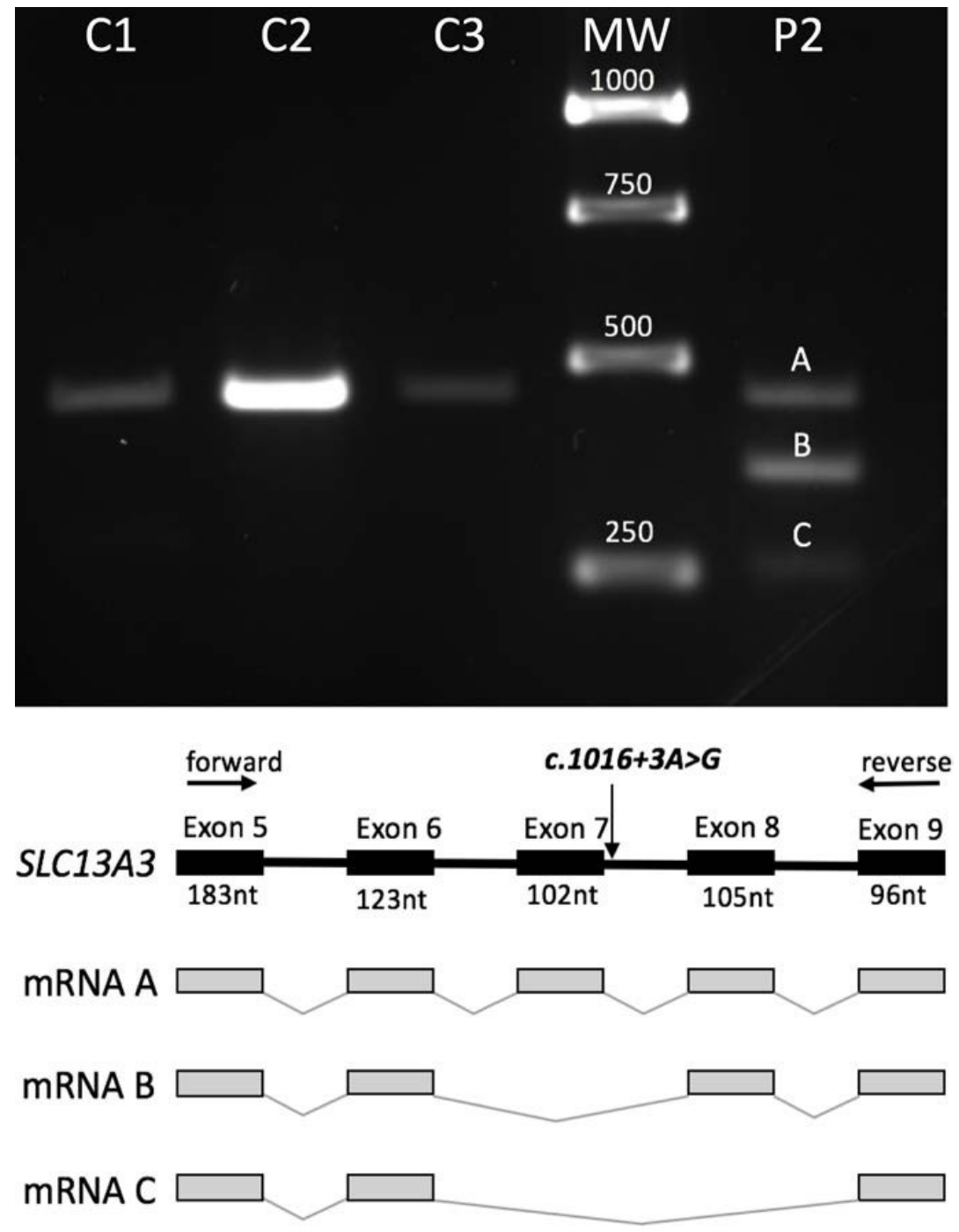

Figure 4 


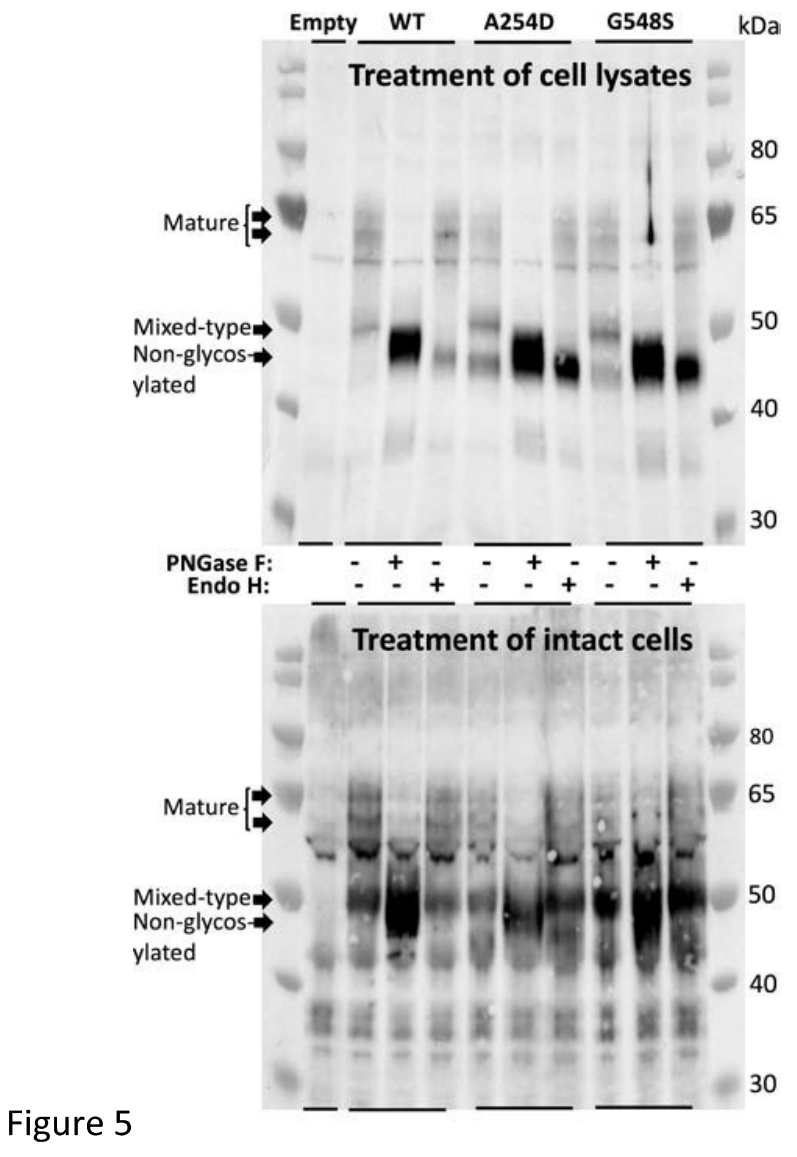

This article is protected by copyright. All rights reserved. 


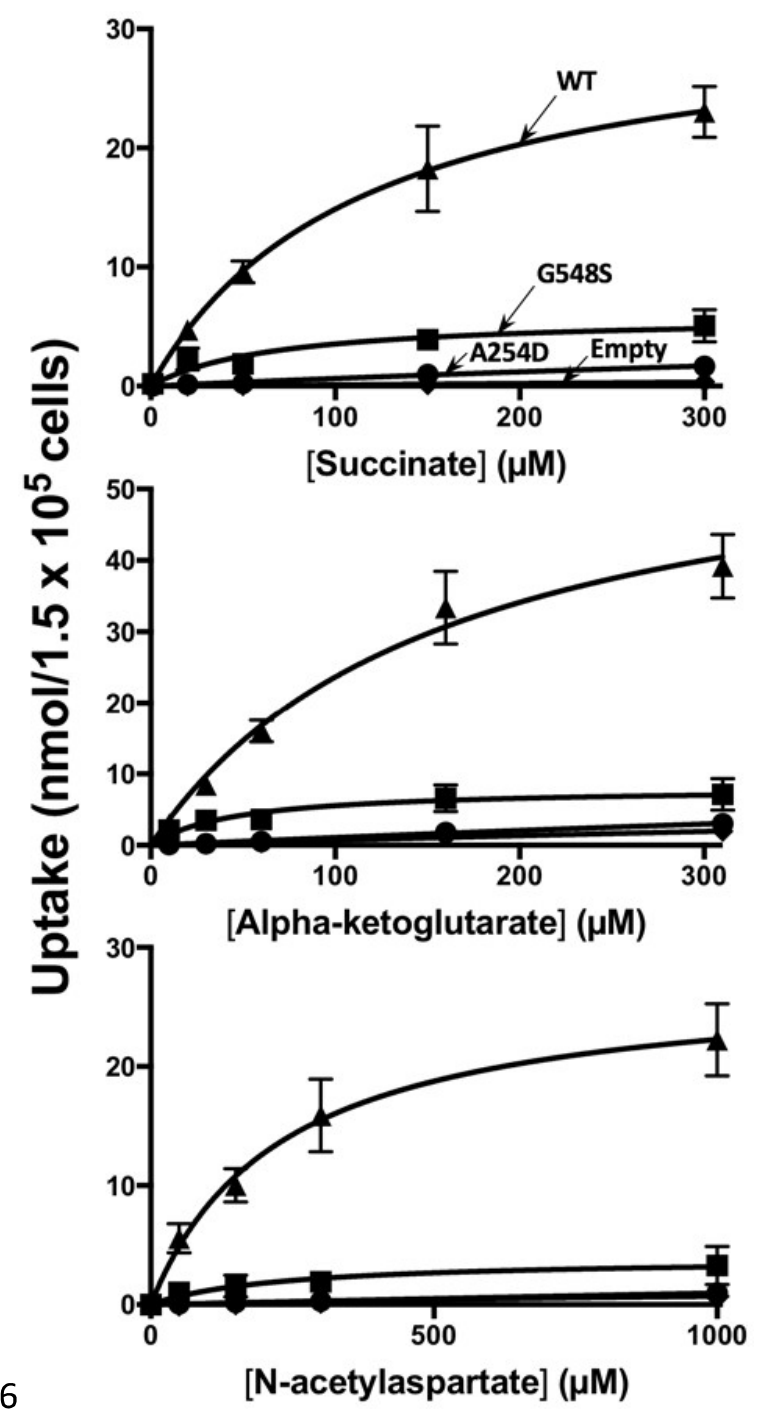

Figure 6 
Figure 7

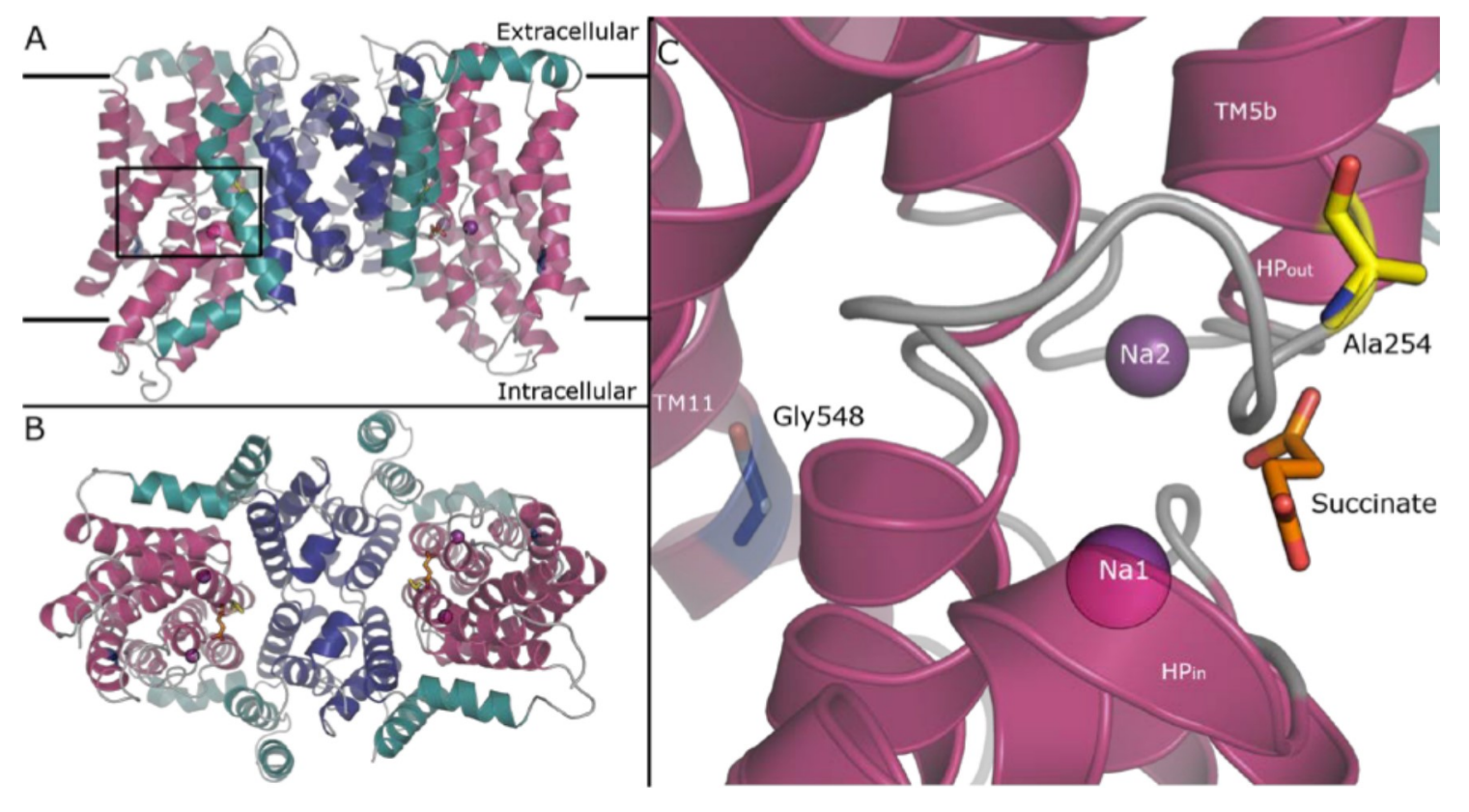

